\title{
Tezacitabine Anhydrous
}

National Cancer Institute

\section{Source}

National Cancer Institute. Tezacitabine Anhydrous. NCI Thesaurus. Code C95916.

The anhydrous form of tezacitabine, a synthetic pyrimidine nucleoside analogue with potential antineoplastic activity. Phosphorylated by cellular kinases, tezacitabine is converted into its active diphosphate and triphosphate metabolites. Tezacitabine diphosphate binds to and irreversibly inhibits the activity of the enzyme ribonucleotide reductase (RNR), which may result in the inhibition of DNA synthesis in tumor cells and eventually tumor cell apoptosis. Tezacitabine triphosphate acts as a substrate for DNA polymerase, thereby further inhibiting DNA replication. RNR catalyzes the conversion of ribonucleoside 5'-diphosphates to deoxyribonucleoside 5'-diphosphates, a necessary step for DNA synthesis, and is overexpressed in many tumor cell types. 